\title{
SEMIPERFECT FPF RINGS
}

\author{
S. S. PAGE
}

\begin{abstract}
In this paper we derive some of the structure of semiperfect FPF rings. A ring is right FPF if every f.g. faithful right module is a generator. For semiperfect right and left FPF rings we show that if all one sided zero divisors are two sided zero divisors, then the classical and maximal quotient rings coincide (all four of them) and are self-injective. We show that if the intersection of the powers of the Jacobson radical is zero, then right and left regular elements are regular. Also, we show right FPF semiperfect rings contain the singular submodule of their injective hulls and that every finitely generated module contained in the injective hull and containing the ring is isomorphic to the ring.
\end{abstract}

Introduction. The objects of study in this paper are semiperfect FPF rings. A ring is right (left) FPF if every finitely generated faithful right (left) module is a generator of the category of all right (left) modules. A ring is FPF if it is both right and left FPF. This notion generalizes the notion of quasi-Frobenius $(\mathrm{QF})$ rings and pseudoFrobenius (PF) rings. The class of right FPF rings is the class for which a weak version of the fundamental theorem of abelian groups holds, namely: A ring $R$ is right FPF if for every finitely generated faithful right module $M, M^{(n)} \cong R \oplus X$ for some integer $n$ and right module $X\left(M^{(n)}\right.$, for $n$ an integer, means the direct sum of $n$ copies of $M$ ). For an extensive study of semiperfect FPF see Faith $[2,3,4]$ and for nonsingular FPF rings see Page $[8,9,10,11]$. Faith $[4]$ was able to describe commutative FPF rings as those commutative rings for which the maximal quotient ring and classical quotient ring coincide, is self-injective, and all finitely generated faithful ideals are projective. We show in this paper that for a semiperfect FPF ring such that right regular and left regular elements are regular that the right and left maximal and classical quotient rings are all the same and self-injective. This generalizes a theorem of Faith [3] for semiperfect Noetherian FPF rings, which states that a Noetherian semiperfect FPF ring is a product of a quasi-Frobenius ring and a semisimple Artinian ring. We also will show under a mild condition that the FPF condition forces right (left) regular elements to be regular. This affords a complete description of a large class of semiperfect FPF rings. Whether this is true in general, i.e. for one sided FPF rings is not known. It is not known if all semiperfect one sided FPF rings are two sided FPF (one sided FPF does not imply two sided FPF in general, nor is the quotient ring self-injective on both sides, see Page [11]).

Received by the editors August 12, 1982 and, in revised form, December 29, 1982. The contents of this paper were given at the Special Session on Ring Theory at the April 1983 meeting of the AMS in Salt Lake City.

1980 Mathematics Subject Classification. Primary 16A51, 16A48, 16A36; Secondary 16A52, 16A08. 
Throughout this paper all rings will have units and all modules, right or left, will be unitary. For a ring $R, J(R)$ will denote the Jacobson radical. For right (left) $R$-modules, $M_{R}\left({ }_{R} M\right), Z_{r}\left(M_{R}\right)\left(Z_{l}\left({ }_{R} M\right)\right)$ will denote the right (left) singular submodule of $M_{R}\left({ }_{R} M\right)$. The symbol $Q_{m}^{r}(R)$ will stand for the maximal right quotient ring of the ring $R$, and $Q_{m}^{l}(R)$ the left maximal quotient ring, $Q_{\mathrm{cl}}^{l}(R)$ the left classical quotient ring, and $Q$ the right injective hull of $R$. We will use $x^{\perp}\left({ }^{\perp} x\right)$ to denote the right (left) annihilator of an element $x$ in a right (left) $R$-module. We will call an element $x \in R$ right (left) regular if $x^{\perp}=0\left({ }^{\perp} x=0\right)$.

Semiperfect FPF rings. A ring $R$ is semiperfect if $R / J(R)$ is semisimple Artinian and idempotents lift from $R / J(R)$ to $R$. We select a complete set of orthogonal primitive idempotents $\left\{e_{i j}\right\}^{k, l_{i}}, i=1, \ldots, k, j=1, \ldots, l_{i}$ such that $1=\sum_{i, j}^{k, l_{i}} e_{i j}, e_{i j} R$ $\cong e_{i h} R$ for all $j, h ; e_{i j} R \not e_{h f} R$ if $i \neq h$ and for each primitive idempotent $e$, $e R \cong e_{i 1} R$ for some $i$. If we form $e_{0}=\sum_{i=1}^{k} e_{i 1}$, then $e_{0} R e_{0}$ is a ring Morita equivalent to $R$ and is called the basic ring of $R$. (It is independent, up to isomorphism of the choices made above.) If $R=e_{0} R e_{0}$ we say $R$ is self-basic and basic rings are self-basic, Faith [5, Chapter 18]. We will maintain the above notation throughout.

A ring is right FPF if every ring Morita equivalent to it is FPF.

The following is found in Faith [2].

THEOREM A. If $R$ is a semiperfect right FPF ring, then for each primitive idempotent $e, e R$ is uniform and if $R$ is self-basic, every nonzero right ideal contains a two sided ideal as a right essential submodule. In particular $R$ has finite uniform, i.e. Goldie dimension.

THEOREM B. If $R$ is a semiperfect right FPF ring, then $R$ is right self-injective if every element of $e J(R) e$ is a left zero divisor, of eRe for every primitive idempotent $e$.

One of the key results for semiperfect right FPF rings is that they have a minimal generator of the category finitely generated right $R$-modules, something like QF-3 rings. Namely, we have,

1.1. THEOREM. If $R$ is a semiperfect right FPF and $M_{R}$ is a finitely generated faithful right $R$-module, then $M_{R} \cong e_{0} R \oplus X_{R}$ for some right $R$-module $X$.

Proof. Let $R$ be a right FPF semiperfect ring. If $M$ is finitely generated and faithful, $M$ must generate $e_{i 1} R$ for each $e_{i 1}$. This says that there is a map $\lambda$, of $M$ to $e_{11} R$ with image $\lambda$ not contained in $e_{i j} J(R)$. Since $e_{11} R / e_{i j} J(R)$ is simple, it follows that $\lambda(m)=e_{11}$ for some $m$ in $M$. So $M \cong e_{i j} R \oplus M_{1}$ for some $M_{1}$. Now $M$ also generates $e_{21} R$. Since for any map $\gamma: M \rightarrow e_{21} R, \gamma\left(e_{11} R\right) \subset e_{21} J$ it must be that $M_{1}$ generates $e_{21} R$. Hence $M \cong e_{11} R \oplus e_{21} R \oplus M_{2}$ for some right $R$-module $M_{2}$. The obvious induction argument finishes the proof.

1.2. CoROllaRY. If $R$ is a self-basic semiperfect ring, then $R$ is right FPF ring iff every faithful finitely generated module $M$ is of the form $R \oplus X$.

2.1. THEOREM. If $R$ is a semiperfect right FPF ring and $M$ is a finitely generated submodule of $Q$ which contains $R$, then $M \cong R$. 
Proof. Let $M$ be as stated in the hypothesis. Now $M$ is faithful so $M=P \oplus X$ where $0 \neq P$ is projective. We know $P \cong \sum_{i=1}^{m} p_{i} R$ where $p_{i} R \cong e_{j} R$ for some $e_{j}$. Choose $P$ so that $m$ is maximal. We claim $X$ contains no projective submodules. Suppose not, then there exists an $x \in X$ so that $x R \cong e_{j i} R$ for some $e_{j i}$ where $e_{j 1} e_{0}=e_{j 1}$. Form $X \oplus\left(e_{0}-e_{j 1}\right) R=N$. Now $N$ is faithful and for any map of $\left(e_{0}-e_{j 1}\right) R$ to $e_{j 1} R$, the image is in $e_{j 1} J$. It follows that $X$ generates $e_{j 1} R$ and hence $X \cong e_{j 1} R \oplus Y$ for some $Y$. This contradicts the maximality of $m$ and establishes the claim. Next write $1=p+x$ with $p \in P$ and $x \in X$. We claim $x \in Z_{r}\left(M_{R}\right)$. To see this note that $e_{i j}=p e_{i j}+x e_{i j}$ for each $i=1, \ldots, k$, and $j=1, \ldots, l_{i}$. Now $x e_{i j} R \not$ $e_{i j} R$ and the kernel of the map $R \rightarrow x e_{i j} R$ given by left multiplication by $x e_{i j}$ is $\left(1-e_{i j}\right) R \oplus W$ where $W \subset e_{i j} J$. Therefore, since $e_{i j} R$ is uniform, this kernel is essential and $x e_{i} \in Z_{r}(M)$ for each $i=1, \ldots, k$, and any $j$. But $x=\sum_{k, j}^{n} x e_{i j}$ so the claim is justified. We now have that $p^{\perp}=0$ since $x^{\perp}$ is essential. This means $p R \cong R$ and that the uniform dimension of $P$ is the same as that of $R$. Of course this implies that $X=0$ and hence that $M=P$ is projective. The next task is to show $P$ is

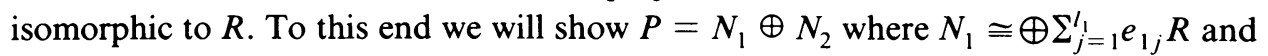
$N_{2}$ is a sum of projective indecomposables none of which is isomorphic to $e_{11} R$. Since $P$ is a generator we know $P \cong e_{11} R \oplus Y$ for some $Y$. Choose $N_{1}=\sum_{j=1}^{m_{1}} p_{j} R$, $p_{j} R \cong e_{11} R$, so that $N_{1} \oplus N_{2}=P$ and $N_{2}$ does not contain a summand isomorphic to $e_{11} R$. We want to show $m_{1} \geqslant l_{1}$. Next notice that

$$
A=\left(\sum_{\substack{h \neq 1 \\ j}} e_{h j} R\right)^{\perp} \neq 0
$$

and is a two ideal contained in $\sum_{j} e_{1 j} R$. To see this $A$ cannot be zero for $\sum_{h \neq 1, j} e_{h j} R$ cannot generate $e_{i 1} R$, by the Krull Schmidt theorem. Also, $A \cap e_{l j_{1}} R=0$ for some $e_{1 j_{1}} R$ but $e_{1 j_{2}} R \cap A \neq 0$ violates the fact that $e_{i j_{1}} R \cong e_{i j_{2}} R$, so $A$ is essential in $\sum_{j} e_{1 j} R$. Now the uniform dimension of $A$ is $l_{1}$. Let $1=n_{1}+n_{2}$ where $n_{1} \in N_{1}$ and $n_{2} \in N_{2} . N_{2} A=0$ for $N_{2}$ is a sum of projectives isomorphic to summands of $\left(e_{0}-e_{11}\right) R$. This gives $A \subset N_{1}$ so the uniform dimension $N_{1} \geqslant l_{1}$. It follows that $m_{1} \geqslant l_{1}$. Notice next that $N_{1}$ cannot generate $e_{2 j} R$, so $N_{2}$ must generate $e_{2 j} R$. But then, as we just have seen, $N_{2}=N_{3} \oplus N_{4}$ where $N_{3} \cong \sum_{j=1}^{m_{2}} p_{2 j} R$ and $m_{2} \geqslant l_{2}$, and $p_{2 j} R \cong e_{21} R$ for all $j$. The obvious induction now gives $P \cong R \oplus X$, but $X=0$ by the uniform dimension argument.

2.2. Corollary. If $R$ is a semiperfect right FPF ring, then for each $q \in Q$, $q Q_{m}^{r}(R)+Q_{m}^{r}(R) \cong Q_{m}^{r}(R)$.

Proof. Trivially $q R+R$ is dense in $q Q_{m}^{r}(R)+Q_{m}^{r}(R)$. But the isomorphism $q R+R \cong R$ lifts to a $Q_{m}^{r}(R)$ isomorphism of $Q$. Also under this extended isomorphism $q$ is sent to an element of $R$ hence of $Q_{m}^{r}(R)$ ard one is also in the image so that the image of $q Q_{m}^{r}(R)+Q_{m}^{r}(R)$ is $Q_{m}^{r}(R)$.

2.3. Corollary. Let $R$ be as in Theorem 2.1. Let $x \in Z_{r}(Q)$. Then $x \in R$. 
Proof. Let $x$ be as stated. We have that $x R+R=d R$ for some $d \in Q$ with $d^{\perp}=0$. Now $d=r_{1}+x r_{2}$ and there is an $r_{3}$ such that $d r_{3}=x$. Since $d^{\perp}=0$, $r_{3}^{\perp}=x^{\perp}$ is essential and $r_{3} \in Z_{r}(R)$ which is contained in $J$ since $Z_{r}(R)$ contains no idempotents. So $x=r_{1} r_{3}+x r_{2} r_{3}$ or $x\left(1-r_{2} r_{3}\right)=r_{1} r_{3}$ and $x=r_{1} r_{3}\left(1-r_{2} r_{3}\right)^{-1} \in R$. Faith [3] calls such rings with $J\left(Q_{m}^{r}(R)\right) \subset R$ sandwich rings.

2.4. CoRollaRY. If $R$ is right FPF semiperfect and $Q=Q_{m}^{r}(R)$, then $J(R) \supset J(Q)$ $=Z_{r}(R)=Z_{r}(Q)$.

PROOF. For a right self-injective ring we have $Z_{r}(Q)=J(Q)$ by Utumi [13, Lemma 4.1].

The next lemma points out the importance of having right regular elements left regular.

3. THEOREM. Let $R$ be a semiperfect right FPF ring. If all right regular elements are left regular, then the regular elements are units in $Q_{m}^{r}(R)$.

Proof. Let $Q$ be the right injective hull of $R$. Let $\Lambda=\operatorname{Hom}_{R}(Q, Q)$. Then $\Lambda$ is a Dedekind finite ring, i.e. $x y=1 \rightarrow y x=1$ in $\Lambda$, since it has no infinite sets of orthogonal idempotents, Jacobson [6]. Let $r \in R$ be such that $r^{\perp}=0$. Then the map $x \rightarrow r x$ induces an isomorphism of $Q$, i.e. a unit of $\Lambda$ which we will denote by $r$ also. So for some $\lambda \in \Lambda$, we have $\lambda \circ r=r \circ \lambda=1$, i.e. $\lambda(r)=\lambda(1) r=1$. Now take $\theta \in \Lambda$ such that $\theta(1)=0$. To show that $\lambda(1)$ is in $Q_{m}^{r}(R)$, we must show that $\theta(\lambda(1))=0$, see Lambeck [7, Proposition 1, p. 94]. We have that $\theta(\lambda(1)) r=0$. Let $\theta(\lambda(1))=y$. Form $y R=R$. Let the embedding of $y R+R$ into $R$ be given by $f$. Then $f(y)$ is in $R$ and $f(y) r=0$, so $f(y)=0$, i.e. $f(\theta(\lambda(1)))=0$ so that $\theta(\lambda(1))=0$. Hence $\lambda(1)$ is $Q_{m}^{r}(R)$ and $r$ is a unit in $Q_{m}^{r}(R)$ which is also Dedekind finite for the same reason $\Lambda$ was.

4.1. Proposition. If $R$ is a semiperfect FPF ring with right regular elements left regular, then $Q_{m}^{r}(R)=Q_{\mathrm{cl}}^{l}(R)=Q$, the right injective hull of $R$.

Proof. We wish to show every element of $Q$ is of the form $a^{-1} b$ for some $a$ and $b$ in $R$ with a regular. Let $q \in Q$, then $q R+R \subset d R$ for some $d$ in $Q$ with $d^{\perp}=0$. But $d R \supset R$ so there exists an element $a$ in $R$ with $d \mathfrak{a}=1$. Now a is right regular so $d=\mathfrak{a}^{-1} \in Q_{m}^{r}(R)$ hence $q \in Q_{m}^{r}(R)$. Also, $q=\mathfrak{a}^{-1} b$ for some $b$ in $R$ which completes the proof.

5. THEOREM. If $R$ is a semiperfect FPF (both sides) ring, with right regular and left regular elements regular, then $Q_{m}^{r}(R)=Q_{m}^{l}(R)=Q_{\mathrm{cl}}^{r}(R)=Q_{\mathrm{cl}}^{l}(R)=Q$ and $Q$ is a right and left self-injective.

Proof. This follows directly from the right and left hand versions of Theorem 3.

REMARK 6. For any ring, $R$, with no infinite sets of orthogonal idempotents with right injective $Q_{m}^{r}(R)$ right regular elements must be left regular so the conditions on regularity are clearly necessary in order that the maximal quotient rings be injective.

Next we show that many semiperfect right FPF rings do have right and left regular elements regular. 
7.1. LEMMA. Let $R$ be a semiperfect right FPF ring. Let $d$ be a right regular element of $R$. Then $\cap_{n=1}^{\infty} R d^{n} \supset^{\perp} d$.

Proof. Let $x d=0$, and form $F=R \otimes R$. Let $M=(d, x) R$ and consider $F / M=$ $N$. We claim $N$ is faithful. To see this suppose $(0,1) r \in M$. Then $(0,1)=(d, x) r_{0}$ for some $r_{0}$. But then $d r_{0}=0$, so $r_{0}=0$ and $x r_{0}=r=0$. This also shows $(0,1) R \cap M=$ 0 . Now $(0,1) R \cong R$ so there is a map $f$ of $N$ into $Q$ such that $f(0,1) R=R$. $N$ is a finitely generated submodule of $Q$ which contains $R$. By Theorem 2.1 we have an epimorphism $\gamma$ of $N$ onto $R$. Now let $\gamma(1,0)=r_{1}$ and $\gamma(0,1)=r_{2}$. Then $R=r_{1} R+$ $r_{2} R$. We claim $r_{1} \in Z_{r}(R) \subset J$. We have that $\left(d^{2}, 0\right) R \subset M$ for $\left(d^{2}, 0\right)=(d, x) d$. This means $r_{1} d^{2}=0$. But since $\left(d^{2}\right)^{\perp}=0, R \cong d^{2} R$. Also $d^{2} R$ is right essential in $R$ because the uniform dimension of $d^{2} R$ is the same as that of $R$. This gives $r_{1} \in Z_{r}(R)$ so, since $r_{1} R$ is small in $R, r_{2} R=R$, hence $r_{2}$ is a unit. Now $r_{1} d+r_{2} x=0$ so $-r_{2}^{-1} r_{1} d=x$ and $x \in R d$. We may repeat the above to $M_{n}=\left(d^{n}, x\right) R$ and $N_{n}=F / M_{n}$ for any $n$ and hence that $x \in R d^{n}$ for all $n$.

7.2. THEOREM. Let $R$ be a semiperfect right and left FPF ring. If for each idempotent $e$, and element $d \in$ eJe such that $d^{\perp} \cap e R e=0$, we have $\cap_{n=1}^{\infty} R d^{n}=0$, then right regular elements are regular.

Proof. If $d$ is right regular and $d$ is right regular modulo the radical $J$, then $d$ is a unit. So we can assume $d$ is not right regular modulo $J$. It follows that there is an idempotent $e$, so that $d e \in J$. Now for primitive idempotents $f$ and $g$, with $f R \cong g R$, if $d f \in J$ then $d g \in J$ too. This means we can take the idempotent $e$ so that $d e \in J$ and if $f$ is a primitive idempotent with $f=(1-e) f=f(1-e)$, then $d f \notin J$, and if $g$ is a primitive idempotent with $g e=e g=g g R \not f R$. This last statement implies that $e d(1-e)$ and $(1-e) d e$ are in $Z_{r}(R) \cap Z_{l}(R)$. Now we have

$$
d=e d e+(1-e) d(1-e)+z
$$

with $z \in Z_{r}(R) \cap Z_{l}(R)$. It follows that ede $+(1-e) d(1-e)$ is a right regular element. It is easy to see that $(1-e) d(1-e)$ is right regular in

$$
(1-e) R(1-e) \text { modulo }(1-e) J(1-e)
$$

since $e d(1-e) \in J$. So $(1-e) d(1-e)$ is a unit in $(1-e) R(1-e)$. Now apply Lemma 7.1 to the right regular element ede $+(1-e) d(1-e)$ and any $y$ such that $y(e d e+(1-e) d(1-e))=0$. This says

$$
\begin{aligned}
y & \in \bigcap_{n=1}^{\infty} R(\text { ede }+(1-e) d(1-e))^{n} \\
& =\bigcap_{n=1}^{\infty} R\left((\text { ede })^{n}+((1-e) d(1-e))^{n}\right) \\
& =\bigcap_{n=1}^{\infty} R((1-e) d(1-e))^{n},
\end{aligned}
$$

in particular that $y e=0$. But for $y=y(1-e), y(1-e) d(1-2) u=y(1-e)$ for some $u \in(1-e) R(1-e)$ and hence that $y=0$. We now have that ede + $(1-e) d(1-e)$ is regular. But since $z \in Z_{l}(R)$, it follows that $d$ is left regular hence regular. 
7.3. Corollary. If $R$ is right FPF and has a.c.c. on left annihilators, then right regular implies left regular.

Proof. Let $d^{\perp}=0$ such that ${ }^{\perp} d$ is maximal. Then Lemma 7.1 implies that if $y d=0$, then $y=r d$ some $r \in R$. But then $r d^{2}=0$ and since ${ }^{\perp} d$ is maximal ${ }^{\perp} d={ }^{\perp}\left(d^{2}\right)$ and hence $r d=y=0$.

7.4. Corollary. If $R$ is left Noetherian right FPF, then $R$ is a left order in a quasi-Frobenius ring.

Proof. By Proposition 4.1 and Corollary $7.3 Q_{\mathrm{cl}}^{l}(R)=Q_{m}^{r}(R)$. This implies $Q_{\mathrm{cl}}^{l}(R)$ is right self-injective and left Noetherian and therefore quasi-Frobenius.

7.5. Corollary. A Noetherian semiperfect FPF ring is a product of Dedekind prime rings and quasi-Frobenius rings.

Proof. By [11] we can decompose an FPF ring into a product $R_{1} \times R_{2}$ with $R_{1}$ semiprime and $R_{2}$ with essential left or right nil ideal. By Theorem 10A of [3] $R_{1}$ is Dedekind. By Corollaries 2.3 and 7.4 and Theorem 2 of [1] $R_{2}$ is Artinian. But Artinian FPF rings are quasi-Frobenius.

7.6. COROLlaRY. If $R$ is right and left FPF and semiperfect, with $\cap_{n=1}^{\infty} R d_{1}^{n}=0=$ $\cap_{n=1}^{\infty} d_{2}^{n} R$ for all right regular $d_{1}$ and left regular $d_{2}$, of eJe in eRe for any idempotent then $Q_{m}^{r}(R)=Q_{m}^{l}(R)=Q_{\mathrm{cl}}^{l}(R)=Q_{\mathrm{cl}}^{r}(R)=Q$.

Proof. This just combines Theorems 5 and 7.2. We obtain a partial converse namely,

8. THEOREM. Let $R$ be a semiperfect ring, with (i) $Q_{m}^{r}(R)=Q_{\mathrm{cl}}^{r}(R)=Q=Q_{\mathrm{cl}}^{l}(R)$, (ii) $Q_{m}^{r}(R)$ is right $F P F$, (iii) $e_{0} R e_{0}$ is strongly bounded, (iv) every finitely generated right ideal of $R$ which contains a regular element is a generator. Then $R$ is right FPF.

Proof. Let $M$ be a finitely generated faithful right $R$-module. We wish to show $M \otimes_{R} Q$ is a faithful $Q$-module. It is easy to see $M \otimes R e_{0}=N$ is a faithful $e_{0} R e_{0}$-module. Now let $\left\{n_{i}\right\}_{i=1}^{k}$ generate $N$ over $e_{0} R e_{0}$. Since $e_{0} R e_{0}$ is strongly bounded $\cap n_{i}^{\perp}=0$. So $e_{0} R e_{0}$ embeds in $N^{(m)}$ for some $m$. We have by [12, Proposition 3.2, p. 219] that $e_{0} Q e_{0}$ is the maximal right ring of quotient of $e_{0} R e_{0}$. We claim $e_{0} Q e_{0}$ is left flat over $e_{0} R e_{0}$. To see this, we know $Q$ is left flat over $R$ because $Q=Q_{\mathrm{cl}}^{r}(R)$ so $I Q=I \otimes Q$ for all right ideals $I$ of $R$. Now each right ideal $H$ of $e_{0} R e_{0}$, is of the form $H=e_{0} I e_{0}$ for a right ideal $I$ of $R$ and

$$
\begin{aligned}
& H \otimes_{e_{0} Q e_{0}} e_{0} Q e_{0} \cong e_{0} I e_{0} \otimes e_{0} Q e_{0} \cong e_{0} I e_{0} \otimes_{e_{0} R e_{0} e_{0} Q \otimes_{e_{0} Q e_{0}} Q e_{0}} \\
& \cong\left(e_{0} I e_{0} \otimes_{e_{0} R e_{0}} e_{0} R\right) \otimes_{R}\left(Q \otimes_{e_{0} Q e_{0}} Q e_{0}\right) \cong\left(e_{0} I e_{0} \otimes e_{0} R \otimes_{R} Q\right) \otimes Q e_{0} \\
& \cong\left(e_{0} I \otimes_{R} Q\right) \otimes_{e_{0} Q e_{0}} Q e_{0} \cong\left(e_{0} I Q\right) \otimes_{e_{0} Q e_{0}} Q e_{0} \cong e_{0} I Q e_{0}
\end{aligned}
$$

so that $e_{0} Q e_{0}$ is left flat oyer $e_{0} R e_{0}$. We have an exact sequence $0 \rightarrow R e_{0} \rightarrow N^{(m)}$. Tensoring this with $e_{p} Q e_{0}$ over $e_{0} R e_{0}$ gives

$$
0 \rightarrow e_{0} Q e_{0} \rightarrow N^{(m)} \otimes e_{0} Q e_{0} \cong\left(M \otimes R e_{0}\right)^{m} \otimes e_{p} Q e_{0} .
$$


So $\left(M \otimes R e_{0}\right) \otimes e_{0} Q e_{0}$ is a faithful $e_{0} Q e_{0}$-module. But then $M \otimes_{R} Q$ is a faithful $Q$-module. Now $M \otimes_{R} Q$ must generate $Q$. So there are maps $f_{i}: M \otimes_{R} Q \rightarrow Q$ so that $\Sigma_{i, j} f_{i}\left(m_{j} \otimes q_{i j}\right)=1$. We have that the image of $M$ in $M \otimes Q$ generates $M \otimes Q$. And we can take the $\left\{m_{j}\right\}$ to generate $M$. Letting $f_{i}\left(m_{j} \otimes 1\right)=b_{i j}^{-1} \mathfrak{a}_{i j}$ and $q_{i j}=c_{i j} d_{i j}^{-1}$ we can find regular $b$ and $d$ so that $b f_{i}\left(m_{j} \otimes 1\right) \in R$ for all $i$ and $j$ and $q_{i j} d \in R$ for all $i$ and $j$. Then each $b f_{i}$ restricted to the image of $M$ in $M \otimes Q$ gives a map of $M$ into $R$ and $b d=\sum b f_{i}\left(m_{j} \otimes q_{i j} d\right)$ so $b d$ is in the trace of $M$ in $R$. By condition (iv) $M$ is a generator.

I would like to thank C. Faith for much of the proof of Theorem 3 and Proposition 4.1 which inspired a good deal of the rest of this paper.

\section{REFERENCES}

1. A. W. Chatters and J. E. Robson, Decomposition of orders in semiprimary ring, Comm. Algebra 8 (1980), 517-532.

2. C. Faith, Injective cogenerator rings and a theorem of Tachikawa. I, II, Proc. Amer. Math. Soc. 60 (1976), 25-30; 62 (1977), 15-18.

3. __ Semiperfect Prüfer and FPF rings, Israel J. Math. 26 (1977), 166-177.

4. Injective quotient rings of commutative rings. II, Lecture Notes Pure Appl. Math., Vol. 72, Dekker, New York, 1982

5. _ Algebra. II. Ring theory, Grundlehren Math. Wiss., Band 191, Springer-Verlag, Berlin, Heidelberg and New York, 1976.

6. N. Jacobson, Some remarks on one-sided inverses, Proc. Amer. Math. Soc. 1 (1950), 352-355.

7. J. Lambeck, Lectures on rings and modules, Blaisdell, New York, 1966; corrected reprint, Chelsea, New York, 1966, 1976.

8. S. Page, Regular FPF rings, Pacific J. Math. 79 (1978), 169-176; correction, 79 (1981), 484-492.

9. __ Semiprime and nonsingular FPF rings, Comm. Algebra 10 (1982), 2253-2260.

10.

11. __ FPF rings and some conjectures of C. Faith, Canad. Math. Bull. (to appear).

12. B. Stenstrom, Rings of quotients, Grundlehren Math. Wiss., Band 217, Springer-Verlag, Berlin, Heidelberg and New York, 1975.

13. Y. Utumi, On continuous rings and self-injective rings, Trans. Amer. Math. Soc. 118 (1965), 158-173.

Department of Mathematics, University of British Columbia, 121-1984 Mathematics Road, Vancouver, British Columbia, Canada 